\title{
Azure Lunulae and Leukoencephalopathy in Wilson Disease
}

\author{
Hiroko Hori, Yosuke Kudo, Yoshiyuki Kuroiwa and Fumiaki Tanaka
}

Key words: Wilson disease, azure lunulae, leukoencephalopathy

(Intern Med 60: 1479, 2021)

(DOI: 10.2169/internalmedicine.5417-20)

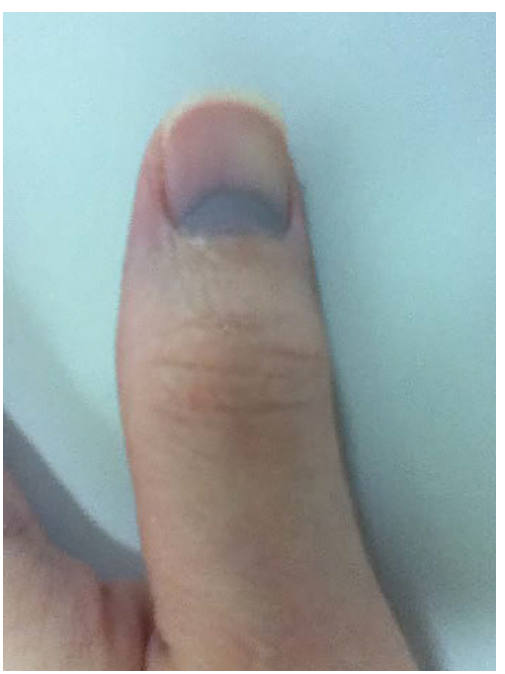

Picture 1.

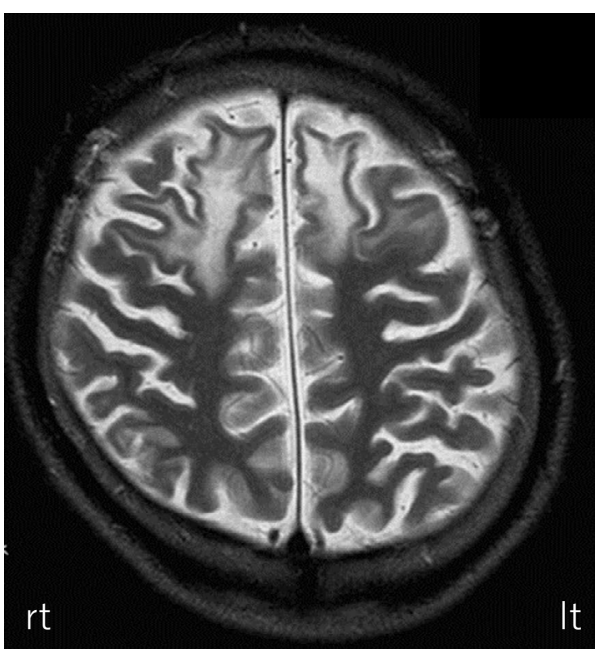

Picture 2.
A 24-year-old Japanese man with no family history of neurological disorder or parental consanguinity developed delusion and extrapyramidal symptoms, including finger tremor and rigidity.

He displayed azure (blue) lunulae of the fingernails (Picture 1). T2 MRI showed high-intensity areas in the frontal white matter (Picture 2). The existence of Kayser-Fleischer rings, low serum ceruloplasmin $(3.1 \mu \mathrm{g} / \mathrm{dL})$, and increased urinary copper excretion $(211 \mu \mathrm{g} / \mathrm{L})$ led to the diagnosis of Wilson disease (WD).

Azure lunulae, which are often reported in argyria, were first described in 1958 as a diagnostic dermatological finding in WD (1); however, given its extreme rarity there are few additional reports.

In WD, frontal and/or parietal white matter MRI lesions have mainly been reported in Asian populations (2); however, isolated white matter lesions without basal nuclei involvement are rare. We should bear in mind that azure lunulae are important diagnostic clues for WD and that isolated white matter changes may occur in WD.

The authors state that they have no Conflict of Interest (COI).

Hiroko Hori , Yosuke Kudo, Yoshiyuki Kuroiwa and Fumiaki Tanaka contributed equally to this work.

\section{References}

1. Bearn AG, McKusick VA. Azure lunulae an unusual change in the fingernails in two patients with hepatolenticular degeneration (Wilson's disease). J Am Med Assoc 22: 904-905, 1958.

2. Takano K, Kuroiwa Y, Shimada Y, Mannen T, Toyokura Y. CT manifestations of cerebral white matter lesion in Wilson disease. Ann Neurol 13: 108-109, 1983.

The Internal Medicine is an Open Access journal distributed under the Creative Commons Attribution-NonCommercial-NoDerivatives 4.0 International License. To view the details of this license, please visit (https://creativecommons.org/licenses/ by-nc-nd/4.0/).

Department of Neurology and Stroke Medicine, Yokohama City University Graduate School of Medicine, Japan Received: May 26, 2020; Accepted: October 18, 2020; Advance Publication by J-STAGE: November 30, 2020 Correspondence to Dr. Fumiaki Tanaka, ftanaka@yokohama-cu.ac.jp

(C) 2021 The Japanese Society of Internal Medicine. Intern Med 60: 1479, 2021 